\title{
Four plasma miRNAs act as biomarkers for diagnosis and prognosis of non-small cell lung cancer
}

\author{
HE-GUO JIANG $^{1 *}$, CHUN-HUA DAI ${ }^{2 *}$, YA-PING XU ${ }^{1}$, QIAN JIANG ${ }^{3}$, XIAN-BIN XIA ${ }^{1}$, YANG SHU ${ }^{3}$ and JIAN LI \\ Departments of ${ }^{1}$ Pulmonary Medicine and ${ }^{2}$ Radiotherapy; ${ }^{3}$ Center of Medical Experiment, \\ Affiliated Hospital of Jiangsu University, Zhenjiang, Jiangsu 212001, P.R. China
}

Received March 19, 2021; Accepted August 26, 2021

DOI: $10.3892 / \mathrm{ol} .2021 .13053$

\begin{abstract}
Previous studies have reported that the aberrant expression of circulating microRNAs (miRNAs/miRs) can be used as diagnostic and prognostic markers in non-small cell lung cancer (NSCLC). The present study aimed to assess the diagnostic and prognostic predictive values of four plasma miRNAs for NSCLC. A total of 12 candidate miRNAs were selected that have previously been reported to be aberrantly expressed in NSCLC, and their plasma levels in the training set were detected via reverse transcription-quantitative PCR analysis. The screened out miRNAs were further validated in the testing set. The area under the curve (AUC) of the receiver operating characteristic curve was constructed to evaluate diagnostic performance. Kaplan-Meier survival analysis was performed to assess the association between the plasma miRNA levels and disease-free survival (DFS) time. The results demonstrated that 4/12 plasma miRNAs (miR-210, miR-1290, miR-150 and miR-21-5p) were highly expressed in patients with NSCLC compared with their expression levels in patients with benign lung disease (BLD) and healthy controls in the training and testing sets, respectively. The AUC values of the four-miRNA panel were 0.96 and 0.93 in the training and testing sets, respectively, for distinguishing patients with NSCLC from healthy controls, which were similar to the AUC values for distinguishing patients with NSCLC from patients with BLD (0.96 and 0.94). The AUC values of the four-miRNA panel in patients with stage I NSCLC were comparable to that of patients with stage II-III NSCLC (0.942 and 0.965). Patients with high plasma levels of miR-210 and miR-150 had worse DFS than those with low plasma levels of these miRNAs. In
\end{abstract}

Correspondence to: Professor Jian Li, Department of Pulmonary Medicine, Affiliated Hospital of Jiangsu University, 438 North Jiefang Street, Zhenjiang, Jiangsu 212001, P.R. China

E-mail: 1ijian541226@163.com

${ }^{*}$ Contributed equally

Key words: non-small cell lung cancer, biomarker, microRNA-210, microRNA-1290, microRNA-150, microRNA-21-5p, diagnosis, prognosis addition, patients whose plasma levels of the four miRNAs decreased by $>50 \%$ after surgery exhibited a good DFS. Taken together, the results of the present study suggest that these four miRNAs (miR-210, miR-1290, miR-150 and miR-21-5p) act as useful biomarkers for early diagnosis and prognosis of NSCLC.

\section{Introduction}

Non-small cell lung cancer (NSCLC) is the main type of lung cancer and has two major histological subtypes, squamous cell carcinoma (SCC) and adenocarcinoma (AD), which accounts for $\sim 85 \%$ of lung cancer cases (1). Due to the late presentation of symptoms and the lack of early diagnosis, numerous patients with NSCLC are diagnosed at advanced stages of the disease (2), which is a major contributing factor to the poor prognosis of NSCLC. Radical surgery remains the most effective treatment for early-stage NSCLC (3). The 5 -year survival rate following surgical excision is $\sim 70 \%$ for patients with stage I NSCLC, and only $30 \%$ for patients with stage III NSCLC (3). Therefore, effective methods of early diagnosis are necessary to reduce the mortality rate. A large, randomized study revealed that screening with low-dose computed tomography (LDCT) for lung cancer results in a $20 \%$ decline in mortality in heavy smokers compared with the findings of the chest X-ray $(4,5)$. However, the high false-positive error, poor cost-effectiveness and the potential side effects associated with LDCT screening limit its use in clinical settings (6). In addition, European research groups have reported less significant results compared with National Lung Screening Trial in the United States and suggested that LDCT scan is not routinely recommended for lung cancer screening (7). Certain protein markers, such as carcinoembryonic antigen (CEA) and cytokeratin 19 fragment 21-1 have been extensively used in clinical practice; however, these tumor markers are not sensitive and specific to contribute to the early detection of NSCLC (8). Thus, the identification of novel markers that can detect the presence of early-stage tumors and predict cancer relapse would provide useful tools for earlier NSCLC diagnosis, with the potential to reduce mortality.

MicroRNAs (miRNAs/miRs) are small non-coding RNAs that modulate gene activity and are aberrantly expressed in the majority of cancer types (9). Circulating miRNAs have 
been demonstrated to serve as biomarkers for cancer diagnosis and prognosis prediction due to their high stability in the bloodstream, cancer-specific regulatory role, reproducibility and ability for non-invasive detection $(10,11)$. Several studies have identified various circulating miRNAs as biomarkers for the diagnosis and prognosis of NSCLC, among numerous differentially expressed miRNAs (12-17). However, due to the difference in research designs, experimental methods and study populations, the results obtained by different groups on this topic markedly vary.

The present study performed reverse transcriptionquantitative PCR (RT-qPCR) analysis to detect a panel of different NSCLC-relevant miRNAs in RNA isolated from the plasma of patients with NSCLC, patients with benign lung disease (BLD) and healthy controls. In the training set, 12 miRNAs (Table SI) that have previously been reported to be associated with NSCLC were selected, and their expression levels in the plasma samples were detected. The significantly aberrant expression of these miRNAs was further evaluated in the testing set. The present study aimed to screen out plasma miRNA expression profiles that may act as valuable biomarkers for the early diagnosis and prognosis of NSCLC.

\section{Materials and methods}

Study design and participants. A total of 128 patients with histopathologically confirmed stage I-IIIA NSCLC, 70 patients with BLD and 60 healthy controls were enrolled in the present study between January 2014 and December 2018 at the Affiliated Hospital of Jiangsu University (Zhenjiang, China). To identify plasma miRNAs as biomarkers for NSCLC, a prospective two-phase and case-control study was designed to distinguish differentially expressed miRNAs between patients with NSCLC and healthy controls. In total, 12 candidate miRNAs that have been reported to be abnormally expressed in NSCLC tissues or blood samples of patients with NSCLC were selected (Table SI) (16-41), and their plasma levels were detected via RT-qPCR analysis in the training set, which was composed of 40 patients with NSCLC and 20 healthy subjects. Next, four miRNAs with significantly different expression levels were selected and determined in the plasma samples of 20 patients with BLD. Subsequently, the validation of these four significantly aberrantly expressed miRNAs in the plasma specimens from the training set was performed in the testing set using plasma samples from 88 NSCLC cases, 50 BLD cases and 40 healthy subjects. These detected miRNAs were compared with serum CEA, a classical tumor marker (8).

The detailed clinical data of the study subjects are summarized in Table I. No significant differences were observed in the distribution of age, sex and smoking status among patients with NSCLC or BLD and healthy controls in the training and testing sets. The patients with NSCLC inclued 55 cases with squamous cell carcinoma (SCC) and 70 cases with non-SCC consisted of 67 cases with adenocarcinoma and 3 cases with large cell carcinoma. All patients with NSCLC underwent tumor resection, and their blood samples were collected prior to surgery, and stored at $-20^{\circ} \mathrm{C}$ until subsequent experimentation. In 88/128 patients with NSCLC, serial blood specimens were collected $\sim 2$ weeks after surgery and before chemotherapy. These 88 patients were followed up from January
2014 to December 2018 (regular outpatient or telephone follow-up every 3 months) and their complete follow-up data were obtained. Analysis of disease-free survival (DFS) was performed according to high or low expression levels of the four plasma miRNAs in the 88 patients, prior to surgery. Subgroup DFS analysis was performed based on histological type. Patients with SCC $(n=41)$ and patients with non-SCC $(n=47)$ were divided into two groups according to high or low expression plasma levels of miR-210 and miR-150 $(n=21$ vs. $n=20$ for miR-210; and $n=24$ vs. $n=23$ for miR-150). In addition, the 88 patients with NSCLC were divided into two groups according to the decreased degree in the plasma levels of the four miRNAs after surgery (>50 and $\leq 50 \%$ ), and survival analysis was performed to assess the impact of the decreased degree in the four plasma miRNA levels on DFS in the patients with NSCLC after surgery. All patients with stage IB-IIIA received platinum-based chemotherapy after surgery. Tumor pathological staging was classified according to the 7th edition of the international tumor-node-metastasis system published by the International Association for the Study of Lung Cancer (42). Blood samples were also collected from patients with BLD and healthy controls and stored at $-20^{\circ} \mathrm{C}$ until subsequent experimentation. The healthy individuals were matched not only with patients with NSCLC, but also with patients with BLD by age, sex and smoking status (Table I). BLD cases included pneumonia $(n=24)$, chronic obstructive pulmonary disease (COPD; $n=20)$, interstitial lung disease $(n=12)$, asthma $(n=8)$ and tuberculous pleurisy $(n=6)$. The present study was approved by the Ethics Review Board of the Affiliated Hospital of Jiangsu University (approval no. 20140019; Zhenjiang, China) and performed in accordance with the Declaration of Helsinki. Written informed consent was provided by all participants prior to the study start.

Plasma preparation and RNA extraction. For plasma preparation, $4 \mathrm{ml}$ venous blood was collected and placed in EDTA-containing tubes (Invitrogen; Thermo Fisher Scientific, Inc.). Whole blood was separated into plasma via centrifugation at $3,000 \mathrm{xg}$ for $10 \mathrm{~min}$ at $4^{\circ} \mathrm{C}$, within $2 \mathrm{~h}$ of collection. Subsequently, $1 \mathrm{ml}$ aliquots of the plasma specimens were transferred into $1.5 \mathrm{ml}$ tubes and centrifuged at 4,000 x g for $10 \mathrm{~min}$ at room temperature to remove any remaining cellular debris. The supernatant was subsequently transferred into clean tubes and stored at $-80^{\circ} \mathrm{C}$ until subsequent experimentation.

Total RNA was isolated from plasma samples using the mirVana PARIS miRNA Isolated kit (Ambion; Thermo Fisher Scientific, Inc.), according to the manufacturer's instructions. The concentration and purity of the extracted RNA were analyzed using a NanoDrop 1000 spectrophotometer (NanoDrop Technologies; Thermo Fisher Scientific, Inc.), and RNA with a concentration $>10 \mathrm{ng} / \mu \mathrm{l}$ was considered acceptable.

$R T$ - $q P C R$. RT-qPCR analysis was performed using the TaqMan MicroRNA RT kit (Applied Biosystems; Thermo Fisher Scientific, Inc.), according to the manufacturer's instructions. Briefly, $5 \mu \mathrm{l}(5-10 \mathrm{ng} / \mu \mathrm{l})$ total RNA was reverse transcribed into cDNA using Avian Myeloblastosis Virus Reverse Transcriptase (Takara Biotechnology Co., Ltd.) and stem-loop RT primers (Takara Biotechnology Co., Ltd.). 
Table I. Demographic and clinical characteristics of subjects in training and testing sets.

\begin{tabular}{|c|c|c|c|c|c|c|}
\hline \multirow[b]{2}{*}{ Characteristic } & \multicolumn{3}{|c|}{ Training set } & \multicolumn{3}{|c|}{ Testing set } \\
\hline & $\begin{array}{l}\text { Healthy controls } \\
\qquad(\mathrm{n}=20)\end{array}$ & $\begin{array}{c}\text { BLD } \\
(n=20)\end{array}$ & $\begin{array}{c}\text { NSCLC } \\
(\mathrm{n}=40)\end{array}$ & $\begin{array}{l}\text { Healthy controls } \\
\qquad(\mathrm{n}=40)\end{array}$ & $\begin{array}{c}\text { BLD } \\
(n=50)\end{array}$ & $\begin{array}{c}\text { NSCLC } \\
(\mathrm{n}=88)\end{array}$ \\
\hline Age, years $($ mean \pm SD) & $58 \pm 9.3$ & $60 \pm 7.8$ & $62 \pm 11.5$ & $59 \pm 10.4$ & $56 \pm 11.5$ & $61 \pm 12.1$ \\
\hline \multicolumn{7}{|l|}{$\operatorname{Sex}, \mathrm{n}(\%)$} \\
\hline Male & $10(50.0)$ & $9(45.0)$ & $19(47.5)$ & $20(50.0)$ & $26(52.0)$ & $47(53.4)$ \\
\hline Female & $10(50.0)$ & $11(55.0)$ & $21(52.5)$ & $20(50.0)$ & $24(48.0)$ & $41(46.6)$ \\
\hline \multicolumn{7}{|l|}{ Smoking status, n (\%) } \\
\hline Ever and current & $8(40.0)$ & $8(40.0)$ & $18(45.0)$ & $15(37.5)$ & $21(42.0)$ & $39(44.3)$ \\
\hline Never & $12(60.0)$ & $12(60.0)$ & $22(55.0)$ & $25(62.5)$ & $29(58.0)$ & $49(55.7)$ \\
\hline \multicolumn{7}{|l|}{ Histology, n (\%) } \\
\hline $\mathrm{SCC}$ & NA & NA & $17(42.5)$ & NA & NA & $41(46.6)$ \\
\hline${ }^{a}$ Non-SCC & NA & NA & $23(57.5)$ & NA & NA & $47(53.4)$ \\
\hline \multicolumn{7}{|l|}{ TNM stage, $\mathrm{n}(\%)$} \\
\hline I & NA & NA & $12(30.0)$ & NA & NA & $23(26.1)$ \\
\hline II & NA & NA & $15(37.5)$ & NA & NA & $40(45.5)$ \\
\hline IIIA & NA & NA & $13(32.5)$ & NA & NA & $25(28.4)$ \\
\hline
\end{tabular}

BLD, benign lung disease; NSCLC, non-small cell lung cancer; SCC, squamous cell carcinoma; TNM, tumor-node-metastasis; NA, not assessed. ${ }^{\mathrm{a}} \mathrm{Non}-\mathrm{SCC}$ included 67 adenocarcinoma and three large cell carcinoma cases.

qPCR was subsequently performed on an Applied Biosystems 7900HT Fast Real-time PCR System (Thermo Fisher Scientific, Inc.) at $90^{\circ} \mathrm{C}$ for $10 \mathrm{~min}$, followed by 40 cycles of $90^{\circ} \mathrm{C}$ for $15 \mathrm{sec}$ and $60^{\circ} \mathrm{C}$ for $1 \mathrm{~min}$. All reactions were performed in triplicate. U6 snRNA was used as the reference gene due to its stability and reproducibly among patients and healthy controls (9-11). The fold change in each miRNA expression relative to U6 snRNA was calculated using the $2^{-\triangle \Delta C q}$ method (43). The expression levels of each target miRNA relative to miRNA expressed in healthy controls were calculated using the $2^{-\Delta \Delta C q}$ method (43). The fold-change of relative miRNA expression was $\log 2$ transformed.

Serum CEA levels were determined using the chemiluminescent microparticle immunoassay ARCHITECT i2000SR (Abbott Pharmaceutical Co. Ltd.), according to the manufacturer's instructions.

Statistical analysis. Statistical analysis was performed using GraphPad Prism 5 (GraphPad Software, Inc.) and SPSS 20.0 software (IBM Corp.). Data are presented as the mean \pm SD from at least three separate experiments. Baseline characteristics of patients with NSCLC or BLD and controls were compared using the Kruskal-Wallis test (age) and Pearson's $\chi^{2}$ or Fisher's exact tests (sex and smoking status). For multiple comparison of the values of miRNA expression levels, the Kruskal-Wallis test was initially used to determine whether the samples originated from the same distribution followed by Steel's-Dwass post hoc test to compare multiple groups with a control. Wilcoxon's test was used to compare miRNA values in paired plasma samples obtained before and 2 weeks after tumor resection.

Receiver operating characteristic (ROC) curve analysis was performed to obtain area under the curve (AUC) values for evaluating diagnostic performance of each plasma miRNA for NSCLC. According to the optimal cut-off values provided by ROC curve analysis, the sensitivity, specificity, and positive and negative predictive values were calculated. Risk scores were assigned to all patients according to a linear combination of the expression levels of the miRNAs in plasma, weighted according to the regression coefficient. Stepwise logistic regression analysis was performed to construct diagnostic miRNA panels based on RT-qPCR results in training and testing setting (44). The prediction probability of being diagnosed with NSCLC from healthy control and BLD or stage I NSCLC from stage II and IIIA NSCLC was used as an index to construct the ROC curve. AUC was used as an index for the diagnostic performance evaluation of the miRNA panels.

To further determine whether the altered miRNAs and other clinical characteristics were independent powerful diagnostic markers for NSCLC, forward stepwise univariate and multivariate logistic regression analyses were performed using the healthy subjects as the reference category. The median value was used as the cut-off to categorize the expression of each miRNA as high or low when assessing their association with disease-free survival (DFS). DFS was defined from the day of surgery to the time of recurrence, mortality or end of follow-up, and was analyzed using the Kaplan-Meier method and log-rank test. $\mathrm{P}<0.05$ was considered to indicate a statistically significant difference.

\section{Results}

Plasma levels of selected miRNAs in patients with NSCLC, patients with BLD and healthy controls. In the initial candidate miRNA selection, a panel of 12 candidate miRNAs that 
have previously been reported to be aberrantly expressed in NSCLC tissues or blood samples of patients with NSCLC (Table SI) (16-41) were analyzed via RT-qPCR analysis in plasma specimens from 40 patients with NSCLC and 20 healthy controls. For the training set, when comparing patients with NSCLC vs. healthy controls, only those miRNAs with a mean fold-change $\geq 2$ and $\mathrm{P}<0.05$ were selected for further analysis in the testing set. The results demonstrated that the expression levels of 4/12 plasma miRNAs, including miR-210, miR-1290, miR-150 and miR-21-5p, were significantly higher in patients with NSCLC compared with those found in the healthy controls $(\mathrm{P}=0.042$ NSCLC compared with healthy control in miR-210; $\mathrm{P}<0.001$ NSCLC compared with healthy control in miR-1290, miR-150 and miR-215p; Table SI and Fig. 1A-D). The expression levels of these four miRNAs were detected via RT-qPCR analysis in 20 patients with BLD, and no significant differences in the expression levels of these miRNAs were observed between patients with BLD and healthy controls (Fig. 1A-D).

These four miRNAs were further evaluated via RT-qPCR analysis in another independent sample set consisting of 88 patients with NSCLC, 50 patients with BLD and 40 healthy controls (testing set). Similar to the results from the training set, the plasma levels of these four miRNAs in patients with NSCLC were significantly higher compared with the healthy controls and patients with BLD $(\mathrm{P}=0.058$, NSCLC compared with BLD and healthy control; $\mathrm{P}<0.001$, NSCLC compared with BLD or healthy control in miR-1290, miR-150 and miR-21-5p; Fig. 2A-D), in the testing set. Similar results were observed for serum CEA levels across the three group in both the training and testing sets (Figs. 1E and 2E).

Diagnostic performance of four plasma miRNAs by ROC curve analysis. ROC curve analyses were performed on the four plasma miRNAs to determine their diagnostic performance for differentiating between patients with NSCLC and healthy controls in the training and testing sets. The results demonstrated that the four miRNAs exhibited similar performance between the two sets, with AUC values of 0.71-0.91 (Fig. 3A and Table SII). When the optimal diagnostic cut-off value of each miRNA was determined via ROC curve analysis, these miRNAs yielded a sensitivity of 70-90\%, a specificity of $70-85 \%$ and an accuracy of 70-88\% (Table SII).

ROC curve analyses were performed in the two sets to distinguish between patients with NSCLC and BLD. The AUC values for these four miRNAs ranged from 0.71-0.91, with a sensitivity of $72-90 \%$, a specificity of $70-86 \%$ and an accuracy of $72-88 \%$ (Fig. 3B and Table SIII). The AUC values for serum CEA in the two sets were $0.69,0.67,0.68$ and 0.65 , respectively, which were inferior to the four aforementioned miRNAs (miR-210, mtR-1290, miR-150 and miR-21-5p) (Fig. 3A and B, and Tables SII and SIII).

To further determine whether the four altered miRNAs and other clinical characteristics are independent powerful diagnostic markers for NSCLC, forward stepwise univariate logistic regression analysis was performed using the healthy controls as the reference category. The results demonstrated that the odds ratios for each of the four miRNAs were statistically significant in the two sets combined (Table SIV). Multivariate logistic regression analysis demonstrated that
miR-1290, miR-150 and miR-21-5p were independently associated with NSCLC after adjusting for age, sex and smoking status (Table SIV).

When the four miRNAs were merged as a panel, they displayed a higher diagnostic performance than that of any individual miRNA alone in distinguishing patients with NSCLC from healthy controls in the training and testing sets, with AUC values of 0.96 and 0.93 , respectively (Fig. 3C and Table SII). The diagnostic performance of the four-miRNA panel was also assessed to distinguish patients with NSCLC from patients with BLD, and the AUC values were 0.96 and 0.94 , respectively, in the two sets (Fig. 3D and Table SIII). However, combining the four miRNAs with CEA failed to further improve the diagnostic performance in any of the two sets (Fig. 3C and D, and Tables SII and SIII).

Subgroup analyses of four plasma miRNAs in patients with NSCLC. The present study assessed the association between the four plasma miRNAs and tumor pathological stage of NSCLC. The results demonstrated that none of the four plasma miRNAs exhibited significantly different expression in patients with stage I NSCLC than those with stage II or IIIA disease (Fig. 4A). The AUC values of the four individual miRNAs in patients with stage I NSCLC (AUCs, 0.71-0.83) were relatively lower than those with stage II NSCLC (AUCs, 0.78-0.86) or stage IIIA NSCLC (AUCs, 0.75-0.92) (Fig. 4B). When combining the four miRNAs as a panel in stage I patients with another panel, including patients with stage II-IIIA disease, the AUC value of the four-miRNA panel was 0.942 to distinguish patients with stage I NSCLC from healthy individuals, with a sensitivity of $90.5 \%$, a specificity of $88.7 \%$ and an accuracy of $89.2 \%$ (Fig. 4C). The AUC value of the four-miRNA panel in differentiating patients with stage II-IIIA NSCLC from healthy individuals was 0.965 , with a sensitivity of $92.4 \%$, a specificity of $90.6 \%$ and an accuracy of $90.4 \%$ (Fig. 4D). Taken together, these results suggest that the four-miRNA signature may possess similar diagnostic performance for patients with stage I and stage II-IIIA NSCLC.

The plasma levels of the four miRNAs in patients with different histological types were analyzed. No significant differences in any of the four miRNAs were observed between patients with SCC and patients with non-SCC (AD and large cell lung cancer) (Kruskal-Wallis test; Fig. 5A). The AUC values of the four miRNAs in patients with SCC were similar to those found in patients without SCC when using the healthy subjects as the control (Fig. 5B).

Association between the four miRNAs and DFS time of patients with NSCLC. To determine whether the plasma levels of the four miRNAs were associated with DFS in patients with NSCLC, 88 patients in the training and testing sets were followed up and the follow-up data were analyzed. The median follow-up time was 42.5 months. The cut-off value for high or low miRNA expression was defined using the median levels of each plasma miRNA. Kaplan-Meier survival analysis demonstrated that high plasma levels of miR-210 and miR-150 were significantly associated with a short DFS time in patients with NSCLC (Fig. 6A and C). However, no significant associations between miR-1290 or miR-21-5p levels and DFS were observed (Fig. 6B and D). 

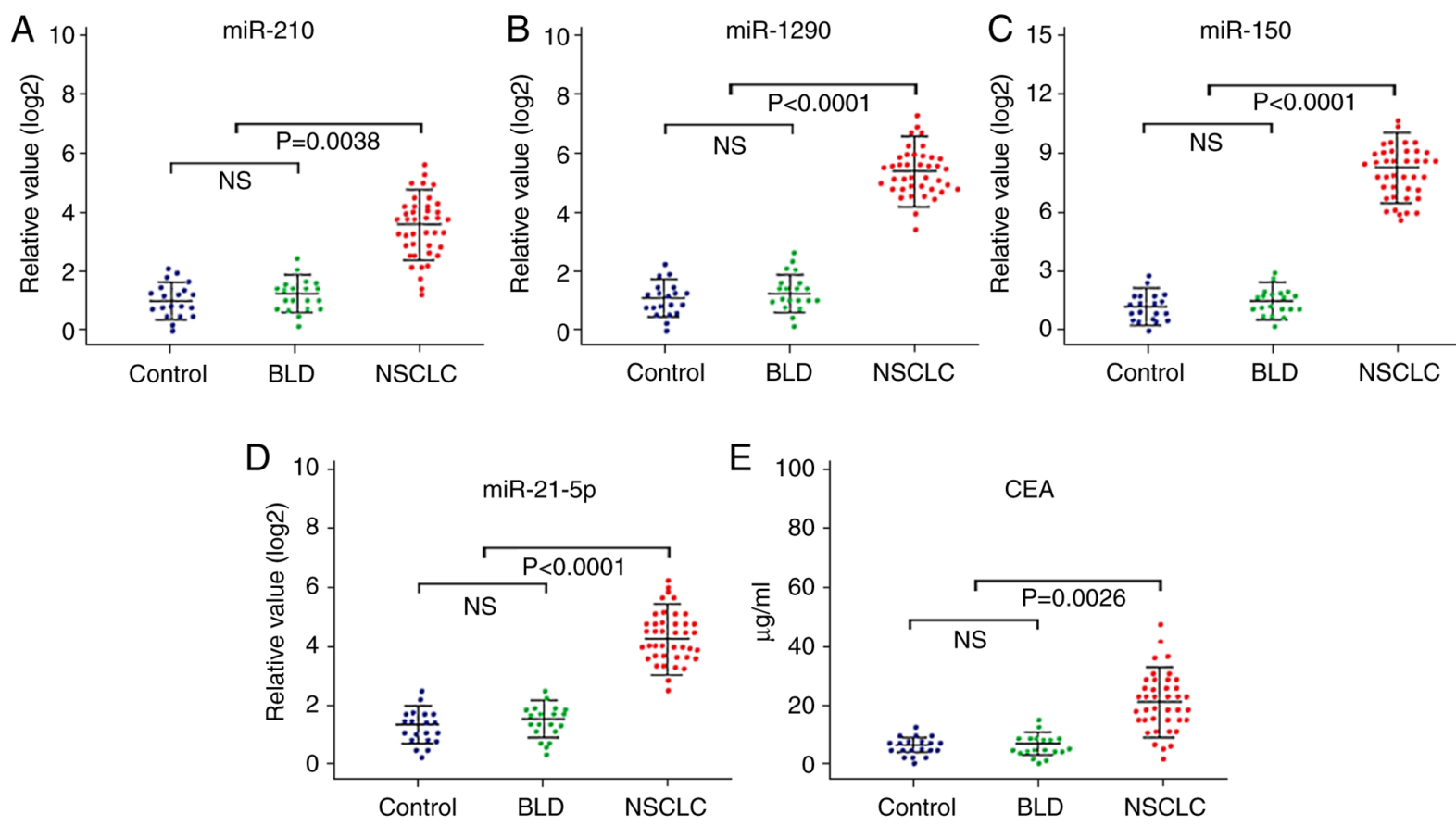

Figure 1. Levels of four plasma miRNAs and serum CEA in the training set. Levels of plasma (A) miR-210, (B) miR-1290, (C) miR-150 and (D) miR-21-5p, and (E) serum CEA in patients with NSCLC or BLD and healthy controls. miRNA/miR, microRNA; CEA, carcinoembryonic antigen; NSCLC, non-small cell lung cancer; BLD, benign lung disease; NS, no significance.
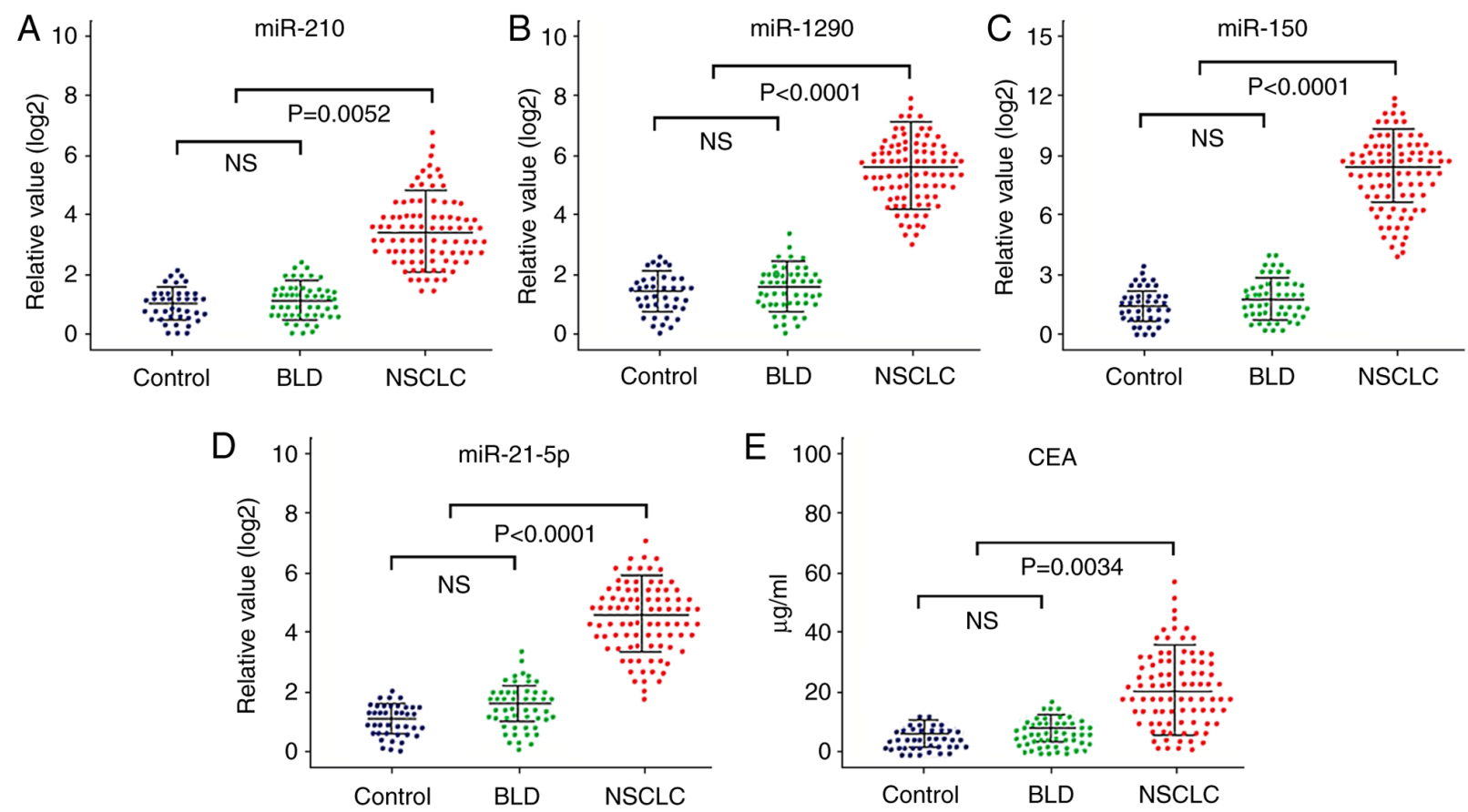

Figure 2. Levels of four plasma miRNAs and serum CEA in the testing set. Levels of plasma (A) miR-210, (B) miR-1290, (C) miR-150 and (D) miR-21-5p, and (E) serum CEA in patients with NSCLC or BLD and healthy controls. miRNA/miR, microRNA; CEA, carcinoembryonic antigen; NSCLC, non-small cell lung cancer; BLD, benign lung disease; NS, no significance.

Given the potential impact of histological type on the DFS of patients with NSCLC who exhibited different plasma levels of miR-210 and miR-150, subgroup analysis was performed to further assess the association between these two miRNAs and DFS in patients with NSCLC. For patients with histologically confirmed non-SCC lung cancer, high plasma levels of miR-210 and miR-150 were associated with shorter DFS times
(Fig. 6F and $\mathrm{H}$ ), whereas the DFS time of patients with SCC was not affected by the plasma levels of miR-210 or miR-150 (Fig. 6E and G).

In the 88 patients with NSCLC, additional plasma samples were collected $\sim 2$ weeks after surgery and before chemotherapy. The results demonstrated that the high pre-operative plasma levels of the four miRNAs significantly decreased after 
A
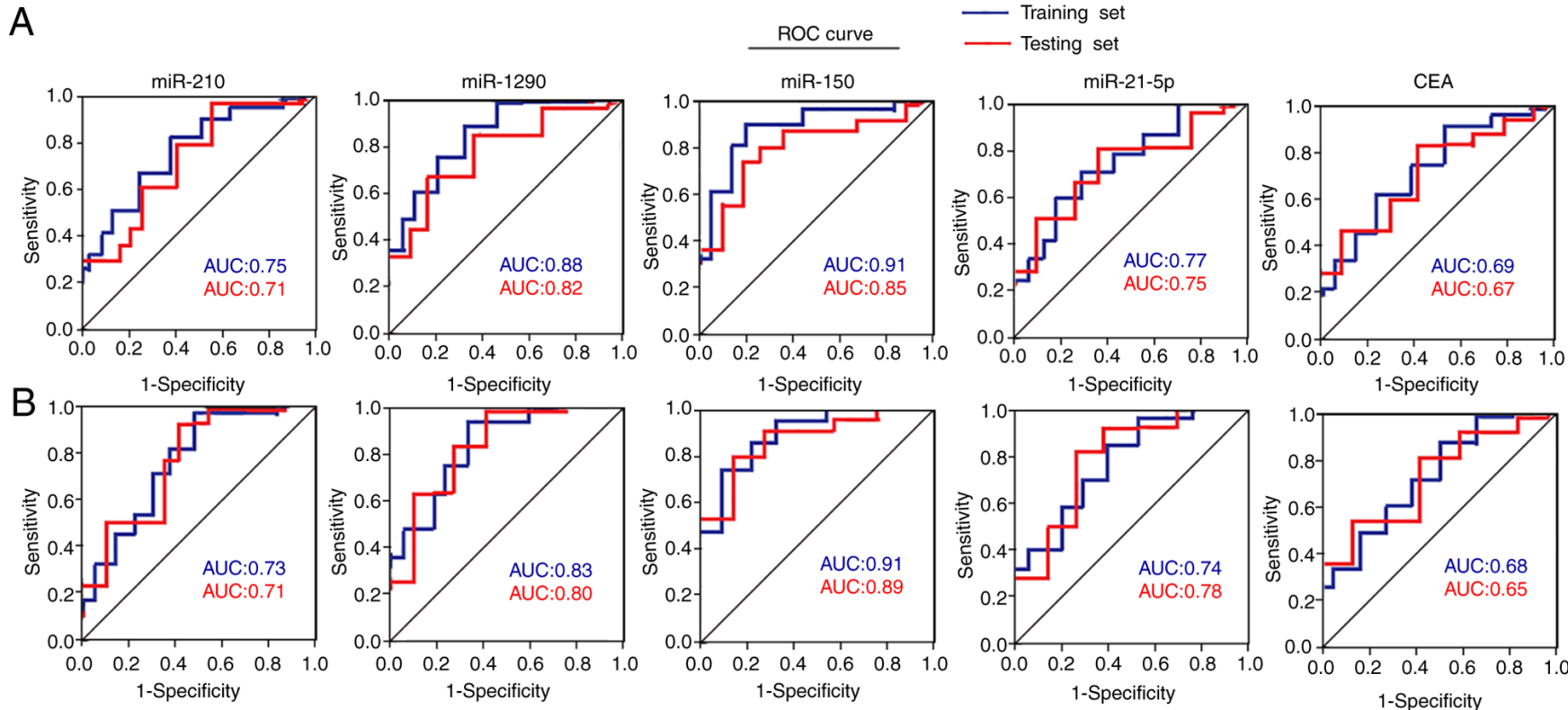

C

ROC curve - Training set
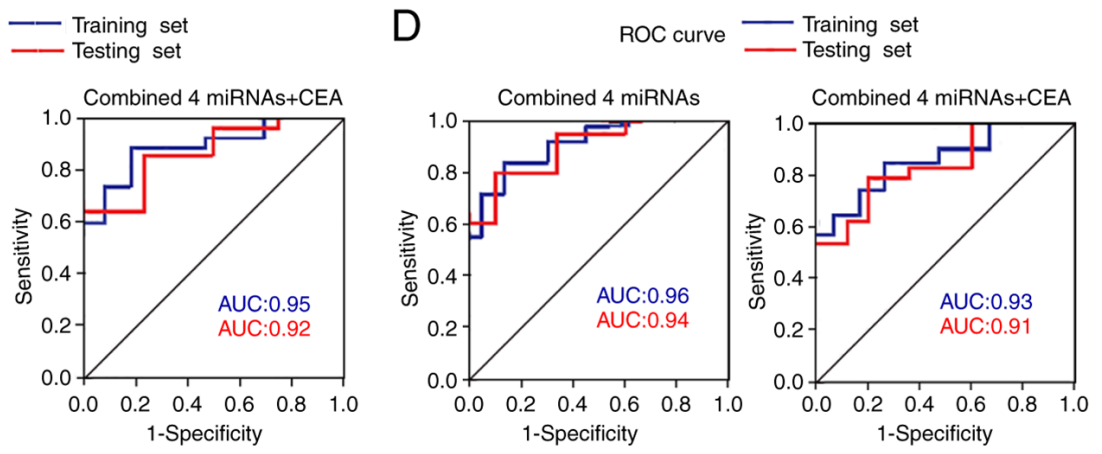

Figure 3. ROC curve analyses in the training and testing sets. ROC curve analyses of plasma miR-210, miR-1290, miR-150, miR-21-5p and CEA levels for distinguishing patients with NSCLC from (A) healthy controls and (B) patients with BLD. ROC curve analyses of the four-miRNA panel and the miRNA panel combined with CEA for distinguishing patients with NSCLC from (C) healthy controls and (D) patients with BLD. ROC, receiver operating characteristic; miRNA/miR, microRNA; CEA, carcinoembryonic antigen; NSCLC, non-small cell lung cancer; BLD, benign lung disease; AUC, area under the curve.

surgery, despite a slight increase in the levels of these four miRNAs in a small number of patients with NSCLC (Fig. 7A). Patients with NSCLC were divided into two groups (>50 and $\leq 50 \%)$ according to the degree of post-operative decrease in the expression levels of the four miRNAs. Survival analysis demonstrated that the DFS time of patients whose plasma levels of the four individual miRNAs decreased by $>50 \%$ after surgery was significantly longer than those with $<50 \%$ reduction in the plasma levels of the four miRNAs (Fig. 7B).

\section{Discussion}

Several circulating miRNAs have been reported to act as diagnostic markers in NSCLC; however, the miRNAs identified by different researchers vary from study to study (12-17). In addition to differences in populations and specimens, variations in the methods used and project design may also result in inconsistencies between these studies. The results of the present study demonstrated that miR-210, miR-1290, miR-150 and miR-21-5P were significantly upregulated in the plasma of patients with NSCLC compared with their expression levels in patients with BLD and healthy controls. The four miRNAs were selected by RT-qPCR analysis from 12 candidate miRNAs that have previously been reported to be aberrantly expressed in NSCLC tissues or blood samples of patients with NSCLC (16-41). The diagnostic value of the four miRNAs for NSCLC was verified via ROC curve analyses in two independent cohorts of age and sex-matched plasma samples (training and testing sets). Univariate and multivariate logistic regression analyses demonstrated the reliability of the diagnostic efficiency of these four miRNAs. Notably, the results of the present study demonstrated that combining the four miRNAs had a higher diagnostic power than that of single miRNAs, and it accurately distinguished patients with NSCLC from patients with BLD and healthy controls.

The diverse and complex molecular events involved in the initiation and development of a malignancy limits the utility of an individual miRNA as a tumor biomarker $(17,19)$. A panel of miRNAs can represent several aspects of carcinogenesis, and the use of these miRNAs in combination can constitute a more complex indicator for NSCLC diagnosis and prognosis than single miRNAs (45). Some miRNAs, such as miR-25 and miR-214, that have been reported in previous studies $(17,28)$, were markedly elevated in the plasma of patients with NSCLC 
A

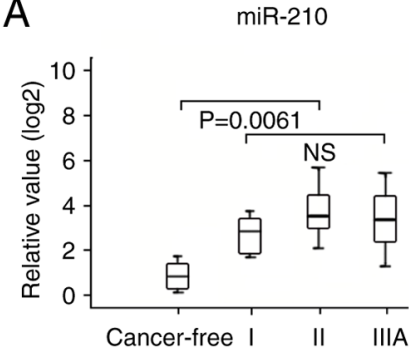

B

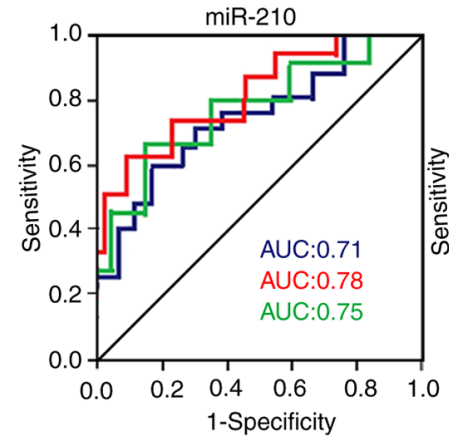

C

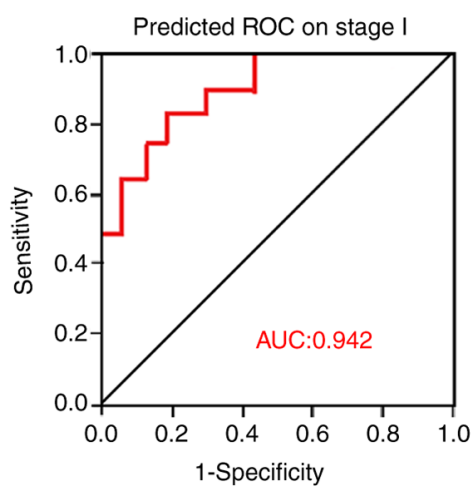

miR-1290

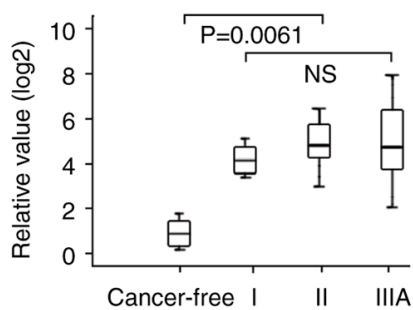

miR-150

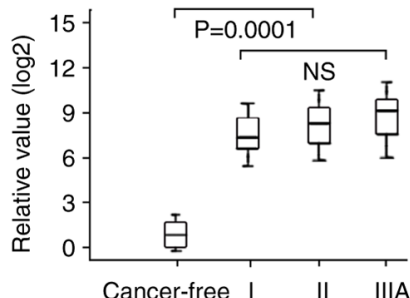

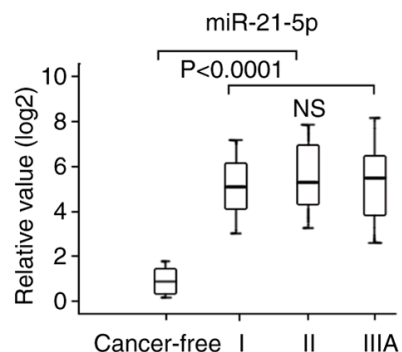

\section{Cancer-free vs stage I \\ - Cancer-free vs stage II}

_ Cancer-free vs stage IIIA
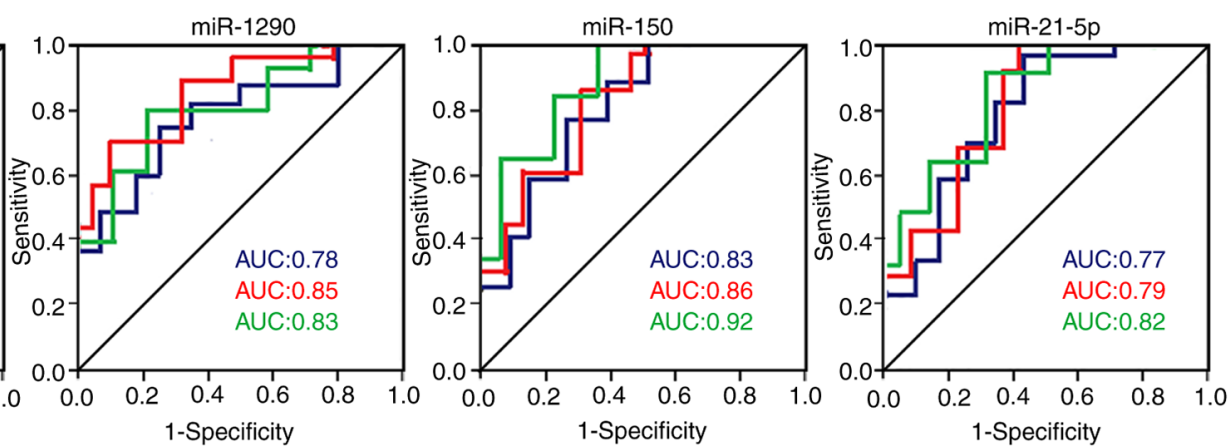

Prediction results (stage I vs Cancer-free)

D

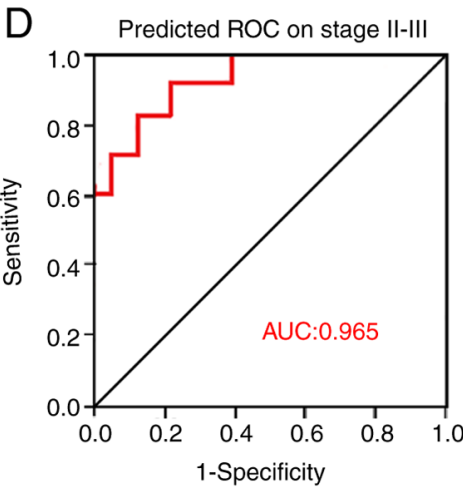

Prediction results (stage II-III vs Cancer-free)
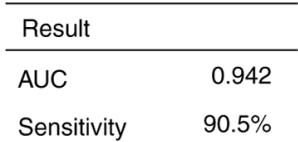

Specificity

$88.7 \%$

PPV

$86.2 \%$

NPV

$82.6 \%$

Accuracy $89.2 \%$

Figure 4. Plasma levels of the four miRNAs in patients with NSCLC with different stages of cancer and healthy controls, and associated ROC curve analyses. (A) The plasma levels of the four miRNAs were significantly upregulated in patients with stage I-IIIA NSCLC compared with the healthy controls. (B) ROC curve analyses were performed on the four individual miRNAs to distinguish patients with NSCLC with different stages (stage I, stage II and stage IIIA) from healthy individuals. ROC curve analysis of the four-miRNA panel in the training and testing sets were combined to distinguish (C) patients with stage I NSCLC or (D) patients with stage II-IIIA NSCLC from healthy individuals, and associated diagnostic performance. miRNA/miR, microRNA; NSCLC, non-small cell lung cancer; ROC, receiver operating characteristic; AUC, area under the curve; PPV, positive predictive value; NPV, negative predictive value.

in the training set in the present study. However, these miRNAs were not assessed in the testing set as they did not meet the selection criteria for further analyses.

The main cause of BLD in the present study included infectious and non-infectious inflammation. Benign disease can lead to various substance changes at the molecular level (46), whereby the microenvironment of the lung is likely to be affected. Abnormal expression of miRNAs occurs in BLD (47-49). Previous studies have reported that several circulating miRNAs are aberrantly expressed in patients with COPD or pneumonia. For example, miR-23a, miR-25, miR-145 and miR-224 are downregulated (47), while miR-29 and miR-126 are upregulated in patients with COPD (48), and miR-193a-5p, miR-542-3p and miR-1246 are markedly elevated in patients with pneumonia, which is associated with disease severity (49). However, no significant differences were observed in the plasma levels of the four miRNAs between patients with BLD and healthy controls in the present study, suggesting that the four plasma miRNAs can be used to distinguish patients with NSCLC from patients with BLD.

The results of the present study demonstrated that the AUC values of the four miRNAs were higher than that of serum CEA levels. Notably, combination of the four miRNAs with CEA did not improve the AUCs of the miRNA-based biomarker for distinguishing patients with NSCLC from the healthy controls, suggesting that the diagnostic performance of the four miRNAs is superior to that of CEA alone, a tumor biomarker widely used in the clinic (50). Notably, the plasma levels of the four miRNAs in patients with stage I NSCLC were markedly elevated compared with the healthy controls and patients with BLD. ROC curve analysis demonstrated that this panel displayed similar diagnostic performance in stage I and stage II-IIIA patients, supporting the four-miRNA panel as a diagnostic marker for early detection of NSCLC. 

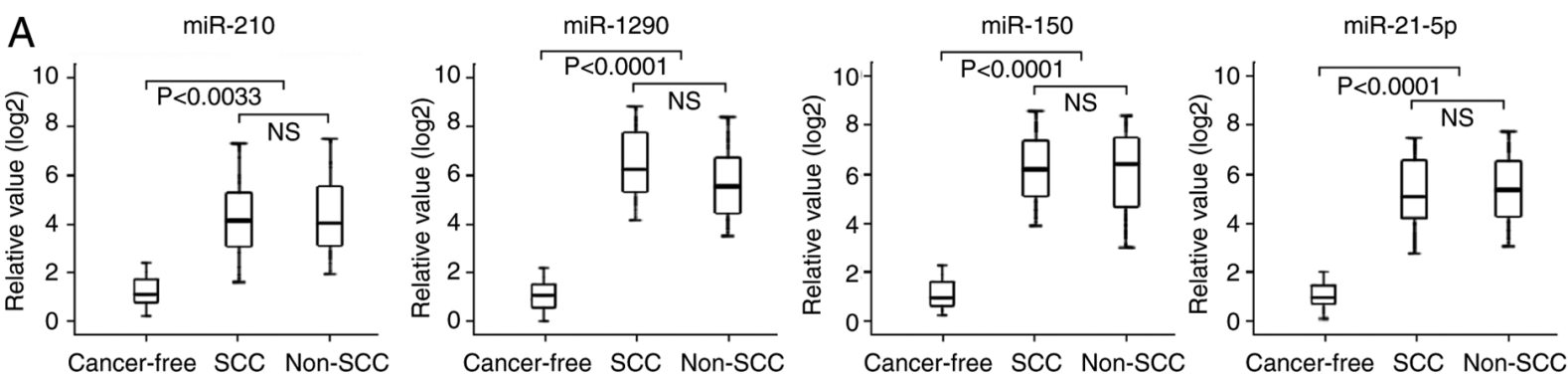

B

ROC curve $\quad$ Cancer-free vs SCC

- Cancer-free vs non SCC
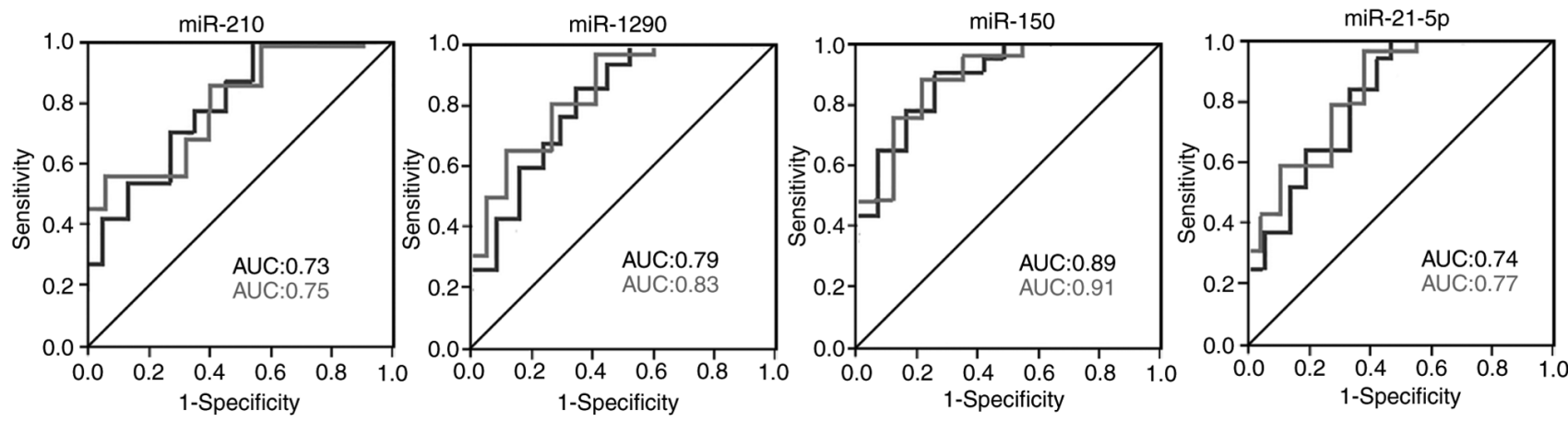

Figure 5. Plasma levels of the four miRNAs in patients with NSCLC with different histological subtypes and healthy controls, and associated ROC curve analyses. (A) The plasma levels of miR-210, miR-1290, miR-150 and miR-21-5p were significantly upregulated in patients with SCC and in patients without SCC (adenocarcinoma and large cell carcinoma) compared with the healthy controls. (B) ROC curve analyses were performed on the four miRNAs combined in the training and testing sets to distinguish patients with NSCLC with different histological subtypes from the healthy controls. miRNA/miR, microRNA; NSCLC, non-small cell lung cancer; ROC, receiver operating characteristic; SCC, squamous cell carcinoma; AUC, area under the curve; NS, no significance.
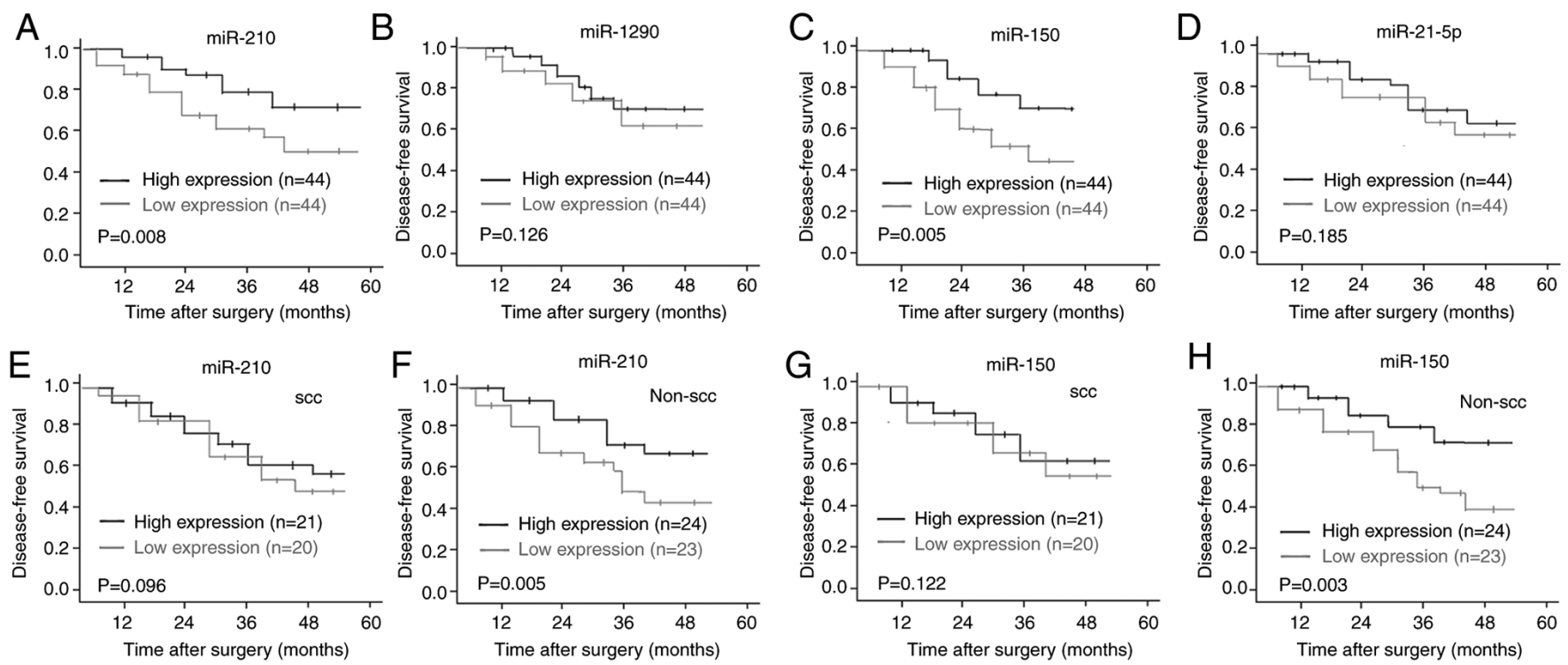

Figure 6. Kaplan-Meier analysis of DFS time in patients with NSCLC according to plasma levels of the four miRNAs. Kaplan-Meier analyses of DFS curves for 88 patients with NSCLC stratified by high or low plasma levels of (A) miR-210, (B) miR-1290, (C) miR-150, and (D) miR-21-5p.. Kaplan-Meier analyses of DFS curves for patients with NSCLC, with different histological subtypes, stratified by high or low plasma levels of (E and F) miR-210 and (G and H) miR-150. DFS, disease-free survival; NSCLC, non-small cell lung cancer; miRNA/miR, microRNA.

Given that patients with NSCLC diagnosed at an early stage can undergo radical surgery of tumors (3), the results of the present study suggest that the use of the four-miRNA panel as a marker for defining early events of NSCLC may be an effective approach to improve prognosis. Histopathological analysis of biopsy tissue is the gold standard for NSCLC diagnosis, which requires invasive methods, such as transthoracic needle puncture or bronchoscopy (12). However, for early-stage NSCLC, it is relatively difficult for a physician to obtain tissue samples by biopsy due to the small lesion size depicted in lung imaging (45). Therefore, it is important to develop novel 
A

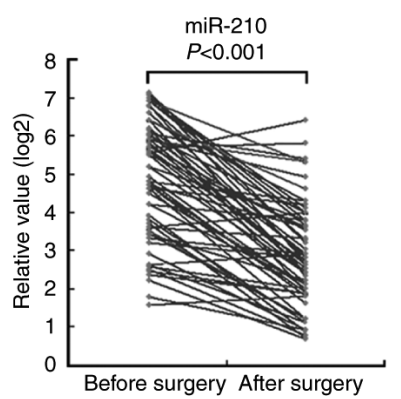

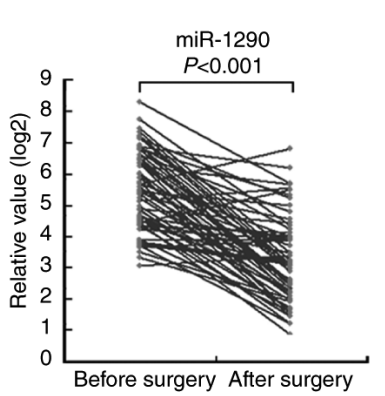
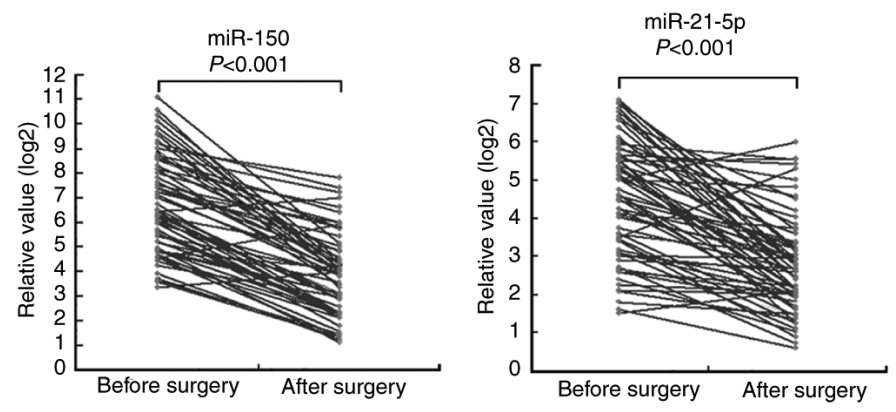

B
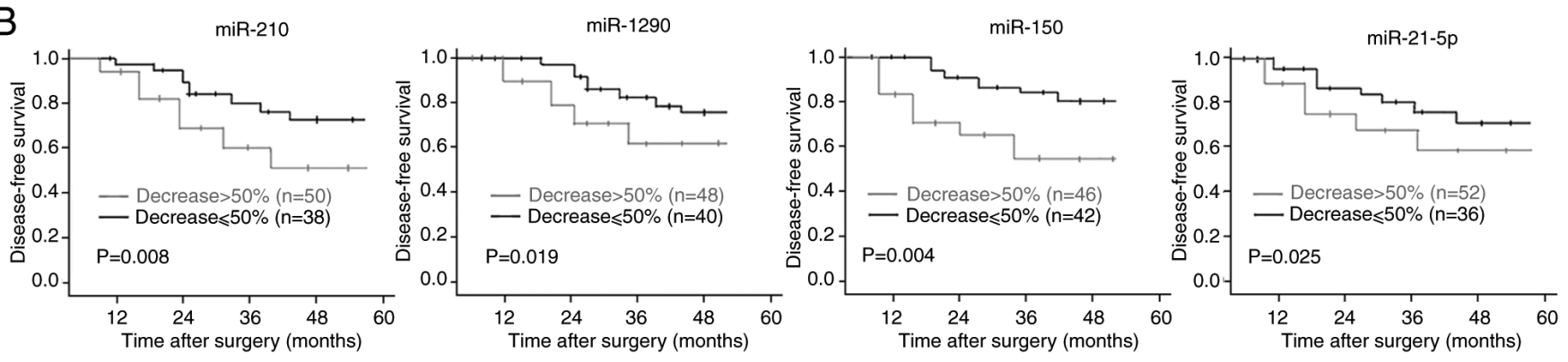

Figure 7. Pre- and post-operative plasma levels of the four miRNAs and their association with DFS in patients with NSCLC. (A) Changes in plasma levels of the four miRNAs in patients with NSCLC before and after surgery. (B) Kaplan-Meier analyses of DFS curves for patients with NSCLC stratified by the degree of decrease in the levels of the four plasma miRNAs after surgery ( $>50 \%$ vs. $\leq 50 \%)$. miRNA/miR, microRNA; DFS, disease-free survival; NSCLC, non-small cell lung cancer.

non-invasive methods and markers with high specificity and sensitivity for the detection of NSCLC.

The present study also assessed the individual role of the four miRNAs in predicting DFS in 88 patients with NSCLC who received surgery and follow-up. The results demonstrated that high plasma levels of miR-210 and miR-150 were associated with shorter DFS time. Notably, high plasma levels of these two miRNAs were significantly associated with a shorter DFS time in patients without SCC lung cancer, but not in patients with SCC. However, no significant differences in the plasma levels of miR-210 or miR-150 were observed between patients with SCC and patients without SCC lung cancer. Furthermore, the results demonstrated an association between DFS and changes in the plasma levels of the four miRNAs before and after surgery. Patients whose plasma levels of the four miRNAs were reduced by $>50 \%$ after surgery had a longer DFS time, suggesting that changes in the pre- and post-operative plasma levels of these four miRNAs may predict prognosis in patients with NSCLC.

Circulating miRNAs can derive from various cell types, including cancer cells, which passively leak or actively transport them into the bloodstream as a way of cell-to-cell communication or an alternative source of circulating miRNAs $(51,52)$. Thus, the deregulation profile, and the diagnostic and prognostic roles of circulating miRNAs may be independent from tissue samples. As circulating miRNAs originate from all cancer cells within an individual, analyzing circulating miRNAs can reduce the impact of the wide heterogeneity of a whole solid tumor compared with miRNAs isolated from a small piece of tissue $(9,10)$. The four aberrantly expressed miRNAs identified in plasma from patients with NSCLC in the present study are well documented NSCLC-related miRNAs. miR-210 is a reliable biomarker for the early diagnosis of NSCLC, which is found in blood samples and sputum and bronchoalveolar lavage fluid samples (53-55). miR-1290 is a tumor-initiating cell-specific miRNA, and together with miR-1246, plays a crucial role in tumor initiation and cancer progression in human NSCLC (56), and it was identified as a potential prognostic biomarker for NSCLC (24). Zhang et al (57) reported that circulating miR-150 can predict prognosis in early-stage NSCLC, and can facilitate cancer cell proliferation by suppressing the tumor suppressor gene, SRC kinase signaling inhibitor 1 . Li et al (58) demonstrated that miR-150-caused autophagy inhibition triggered endoplasmic reticulum stress, increased cellular reactive oxygen species levels, activated the DNA damage response and facilitated NSCLC cell proliferation and tumor growth. miR-21-5p upregulation in tumor samples has been observed in patients with NSCLC, and has been confirmed as an independent prognostic predictor for overall survival (59). Another study reported that miR-21-5p expression in NSCLC tissue is associated with histological subtype, tumor volume, regional lymph node and distal metastasis, and that miR-21-5p promotes the progression of NSCLC by modulating SMAD7 expression (60).

The four miRNAs in blood samples of other types of cancer have also been assessed. For example, circulating miR-210 is significantly increased in patients with breast cancer and hepatocellular carcinoma $(61,62)$. Furthermore, serum miR-1290 is markedly overexpressed in patients with pancreatic cancer and ovarian cancer $(63,64)$. Circulating miR-150 is upregulated in patients with colorectal cancer (65), and serum miR-21-5p is significantly elevated in patients with advanced papillary renal cell carcinoma (66). Taken together, these results suggest that the four miRNAs are involved in the occurrence and development of other cancers, and may be used as biomarkers for these cancers.

The present study is not without limitations. First, the total sample size was relatively small, which may have resulted in 
bias. Furthermore, the follow-up time of patients after surgery was not long enough, and only a small number of patients were included. Furthermore, the impact of hemolysis on the expression of miRNAs was not evaluated by measuring the hemolysis grade of the plasma samples, which may influence the diagnostic accuracy of the four plasma miRNAs for NSCLC.

In conclusion, the results of the present study demonstrated the potential of a four-plasma miRNA signature in the detection of early-stage NSCLC. The results also indicated that the plasma expression profiles of miR-210 and miR-150 can act as prognostic biomarkers for patients with NSCLC, mainly for those with the lung AD subtype. In addition, a significant decrease in the levels of these four plasma miRNAs ( $>50 \%$ ) after surgery is a predictive factor of a longer DFS time. However, prospective cohorts are required to validate the results presented here.

\section{Acknowledgements}

Not applicable.

\section{Funding}

The present study was supported by research grants from the Medical Research Program of Jiangsu Health Committee in China (grant no. ZDB2020022) and the Social Development Foundation of Zhenjiang in China (grant nos. SH2014076 and SH2015063).

\section{Availability of data and materials}

The datasets used and/or analyzed in the current study are available from the corresponding author on reasonable request.

\section{Authors' contributions}

JL, HGJ and CHD conceived and designed the present study. YPX, XBX, HGJ, CHD, QJ and YS performed the experiments, and analyzed and interpreted the data. QJ and YS confirm the authenticity of all the raw data. HGJ and $\mathrm{JL}$ were involved in project development, data analysis and editing the manuscript. All authors have read and approved the final manuscript.

\section{Ethics approval and consent to participate}

The present study was approved by the Ethics Review Board of the Affiliated Hospital of Jiangsu University (approval no. 20140019; Zhenjiang, China) and performed in accordance with International Ethical Guidelines for Biomedical Research Involving Human Subjects (CIOMS) (67) and the Declaration of Helsinki of 1964 (68) and a later version (69). Written informed consent was provided by all participants prior to the study start.

\section{Patient consent for publication}

Not applicable.

\section{Competing interests}

The authors declare that they have no competing interests.

\section{References}

1. Travis WD, Brambilla E, Nicholson AG, Yatabe Y, Austin JHM, Beasly MB, Chirieac LC, Dacic S, Duhig E, Flieder DB, et al: The 2015 world health organization classification of lung tumors: Impact of genetic, clinical and radiologic advances since the 2004 classification. J Thorac Oncol 10: 1243-1260, 2015.

2. Chen W, Zheng R, Baade PD, Zhang S, Zeng H, Bray F, Jemal A, Yu XQ and He L: Cancer statistics in China, 2015. CA Cancer J Clin 66: 115-132, 2016.

3. Mominioni L, Imperatori A, Rovera F, Ochetti A, Torrigiotti G and Paolucci M: Stage I nonsmall cell lung carcinoma: Analysis of survival and implications for screening. Cancer 89 (11 Suppl): S2334-S2344, 2000

4. National Lung Screening Trial Research Team, Aberle DR, Adams AM, Berg CD, Black WC, Clapp JD, Fagerstrom RM, Gareen IF, Gatsonis C, Marcus PM and Sicks JD: Reduced lung-cancer mortality with low-dose computed tomographic screening. N Engl J Med 365: 395-409, 2011.

5. National Lung Screening Trial Research Team, Church TR, Black WC, Aberle DR, Berg CD, Clingan KL, Duan F, Fagerstrom RM, Gareen IF, Gierada DS, et al: Results of initial low-dose computed tomographic screening for lung cancer. $\mathrm{N}$ Engl J Med 368: 1980-1991, 2013.

6. Snowsill T, Yang H, Griffin E, Long L, Varley-Campbell J, Coelho H, Robinson S and Hyde C: Low-dose computed tomography for lung cancer screening in high risk populations: A systematic review and economic evaluation. Health Technol Assess 22: 1-276, 2018.

7. Veronesi G: Lung cancer screening: The European perspective. Thorac Sury Clin 25: 161-174, 2015.

8. Okarnura K, Takayama K, Izumi M, Harada T, Furuyama K and Nakanish Y: Diagostic value of CEA and CYFRA 21-1 tumor markers in primary lung cancer. Lung Cancer 80: 45-49, 2013.

9. Iorio MV and Croce CM: MicroRNA dysregulation in cancer: Diagnosis, monitoring and therapeutics-A comprehensive review. EMBO Mol Med 4: 143-159, 2012.

10. Pardini B, Sabo AA, Birolo G and Calin GA: Noncoding RNAs in extracellular fluids as cancer biomarkers: The new frontier of liquid biopsies. Cancers (Basel) 11: 1170, 2019.

11. Larrea E, Sole C, Manterola L, Goicoechea I, Armesto M, Arestin M, Caffarel MM, Araujo AM, Araiz M, Fernandez-Mercado $\mathrm{M}$ and Lawrie $\mathrm{CH}$ : New concepts in cancer biomarkers: Curculating miRNA in liquied biopsies. Int J Mol Sci 17: 627, 2016.

12. Chen X, Hu Z, Wang W, Ba Y, Ma L, Zhang C, Wang C, Ren Z, Zhao Y, Wu S, et al: Identification of ten serum microRNAs from a genome-wide serum micreRNA expression profile as novel noninvasive biomarkers for nonsmall cell lung cancer diagnosis. Int J Cancer 130: 1620-1628, 2012.

13. Nadal E, Truini A, Nakata A, Lin J, Reddy RM, Chang AC, Ramnath N, Gotoh N, Beer DG and Chen G: A novel serum 4-microRNA signature for lung cancer detection. Sci Rep 5: 12464, 2015.

14. Lv S, Xue J, Wu C, Wang L, Wu J, Xu S, Liang X and Lou J: Identification of a panel of serum microRNAs as biomarkers for early detection of lung adenocarcinoma. J Cancer 8: 48-56, 2017.

15. Shang AQ, Xi YN, Wang J, Sun L, Wei J, Lu WY, Lan JY, Wang WW, Wang L and Wang LL: Predictive value of serum microRNA-22 and microRNA-126 levels for non-small cell lung cancer development and metastasis: A case-control study. Neoplasma 64: 453-459, 2017.

16. Foss KM, Sima C, Ugolini D, Neri M, Allen KE and Weiss GJ: MiR-1254 and miR-574-5P: Serum-based microRNA biomarkers for early-stage non-small cell lung cancer. J Thorac Oncol 6: 482-488, 2011.

17. Wang C, Ding M, Xia M, Chen S, Van Le A, Soto-Gil R, Shen Y, Wang $\mathrm{N}$, Wang $\mathrm{J}, \mathrm{Gu} \mathrm{W}$, et al: A Five-miRNA panel identified from a multicentric case-control study serves as a novel diagnostic tool for ethnically diverse non-small-cell lung cancer patients. EBioMedicine 2: 1377-1385, 2015. 
18. Tan X, Qin W, Zhang L, Hang J, Li B, Zhang C, Wan J, Zhou F, Shao K, Sun Y, et al: A 5-microRNA signature for lung squamous cell carcinoma diagnosis and hsa-miR-31 for prognosis. Clin Cancer Res 17: 6802-6811, 2011

19. Guan P, Yin Z, Li X, Wu W and Zhou B: Meta-analysis of human lung cancer microRNA expression profiling studies comparing cancer tissue with normal tissue. J Exp Clin Cancer Res 31: 54 2012

20. Yang C, Sun C, Liang X, Xie S, Huang J and Li D: Integrative analysis of microRNA and mRNA expression profiles in non-small-cell lung cancer. Cancer Gene Ther 23: 90-97, 2016.

21. Eilertsen M, Andersen S, Al-Saad S, Richardsen E, Stenvold H Hald SM, Al-Shibli K, Donnem T, Busund LT and Bremnes RM: Postive prognostic impact of miR-210 in non-small cell lung cancer. Lung Cancer 83: 272-278, 2014.

22. Duncavage E, Goodgame B, Sezhiyan A, Govindan R and Pfeifer J: Use of microRNA expression levels to predict outcomes in resected stage I non-small cell lung cancer. J Thorac Oncol 5 : $1755-1760,2010$

23. Cao M, Hou D, Liang H, Gong F, Wang Y, Yan X, Jiang X, Wang $\mathrm{C}$, Zhang J, Zen $\mathrm{K}$, et al: MiR-150 promotes the proliferation and migration of lung cancer cells by targeting SRC kinase signalling inhibitor 1. Eur J Cancer 50: 1013-1024, 2014.

24. Mo D, Gu B, Gong X, Wu L, Wang H, Jiang Y, Zhang B, Zhang M, Zhang Y, Xu J and Pan S: MiR-1290 is a potential prognostic biomarker in non-small cell lung cancer. J Thorac Dis 7: 1570-1579, 2015

25. Liang B, Wang GX, Long G, Qiu JH and Hu ZL: Tumor suppressor miR-22 suppresses lung cancer cell progression through post-transcriptional regulation of ErbB3. J Cancer Res Clin Oncol 138: 1355-1361, 2012.

26. Shin YM, Yun J, Lee OJ, Han HS, Lim SN, An JY, Lee KH, Lee $\mathrm{KM}$ and Choe $\mathrm{KH}$ : Diagnostic value of circulating extracellular miR-134, miR-183, and miR-22 levels in lung adenocarcinoma-associated malignant pleural effusion. Cancer Res Treat 46: 178-185, 2014.

27. Wu T, Chen W, Kong D, Li X, Lu H, Liu S, Wang J, Du L, Kong Q, Huang X and Lu Z: MiR-25 targets the modulator of apoptosis 1 gene in lung cancer. Carcinogenesis 36: 925-935, 2015 .

28. Wang P, Yang D, Zhang H, Wei X, Ma T, Cheng Z, Hong Q, Hu J, Zhuo H, Song Y, et al: Early detection of lung cancer in serum by a panel of microRNA biomarkers. Clin Lung Cancer 16: 313-319. e1, 2015.

29. Heegaard NH, Schetter AJ, Welsh JA, Yoneda M, Bowman ED and Harris CC: Circulating miroRNA expression profiles in early stage non-small cell lung cancer. Int J Cancer 130: 1378-1386, 2012

30. Zhu W, He J, Chen D, Zhang B, Xu L, Ma H, Liu X, Zhang Y and Le H: Expression of miR-29c, miR-93, and miR-429 as potential biomarkers for detection of early stage non-small lung cancer. PLoS One 9: e87780, 2014

31. Boeri M, Verri C, Conta D, Roz L, Modena P, Facchinetti F, Calabrò E, Croce CM, Pastorino U and Sozzi G: MicroRNA signatures in tissues and plasma predict development and prognosis of computed tomography detected lung cancer. Proc Nat Acad Sci USA 108: 3713-3718, 2011.

32. Patnaik SK, Yendamuri S, Kannisto E, Kucharczuk JC, Singhal S and Vachani A: MicroRNA expression profiles of whole blood in lung adenocarcinoma. PLoS One 7: e46045, 2012.

33. Long H, Wang Z, Chen J, Xiang T, Li Q, Diao X and Zhu B MicroRNA-214 promotes epithelial-mesenchymal transition and metastasis in lung adenocarcinoma by targeting the suppressor-of-fused protein (Sufu). Oncotarget 6: 38705-38718, 2015.

34. Kim G, An HJ, Lee MJ, Song JY, Jeong JY, Lee JH and Jeong HC: Hsa-miR-1246 and hsa-miR-1290 are associated with stemness and invasiveness of non-small cell lung cancer. Lung Cancer 91: $15-22,2016$

35. Rani S, Gately K, Crown J, O'Byrne K and O'Driscoll L: Global analysis of serum microRNAs as potential biomarkers for lung adenocarcinoma. Cancer Biol Ther 14: 1104-1112, 2013

36. Lu S, Kong H, Hou Y, Ge D, Huang W, Ou J, Yang D, Zhang L, Wu G, Song Y, et al: Two plasma microRNA panels for diagnosis and subtype discrimination of lung cancer. Lung Cancer 123 : 44-51, 2018.

37. Huang W, Li H and Luo R: The microRNA-1246 promotes metastasis in non-small cell lung cancer by targeting cytoplasmic polyadenylation element-binding protein 4. Diagn Pathol 10: 127, 2015 .
38. Zhang N, Wei $\mathrm{X}$ and $\mathrm{Xu} \mathrm{L}$ : MiR-150 promotes the proliferation of lung cancer cells by targeting P53. FEBS Lett 587: 2346-2351, 2013

39. Yin QW, Sun XF, Yang GT, Li XB, Wu MS and Zhao J: Increased expression of microRNA-150 is associated with poor prognosis in non-small cell lung cancer. Int J Clin Exp Pathol 8: 842-846, 2015.

40. Tian F, Li R, Chen Z, Shen Y, Lu J, Xie X and Ge Q: Differentially expressed miRNAs in tumor, adjacent, and normal tissues of lung adenocarcinoma. Biomed Res Int 2016: 1428271, 2016.

41. Ma R, Wang C, Wang J, Wang D and Xu J: MiRNA-mRNA interaction network in non-small cell lung cancer. Interdiscip Sci 8: 209-219, 2016.

42. Goldstraw P (ed): Staging Manual in Thoracic Oncology. Orange Pakk, FL: Editorial Rx Press, pp57-65, 2009.

43. Livak KJ and Schmittgen TD: Analysis of relative gene expression data using real-time quantitative PCR and the 2(-Delta Delta C(T) method. Methods 25: 402-408, 2001.

44. DeLong ER, DeLong DM and Clarke-Pearson DL: Comparing the area under two or more correlated receiver operating characteristic curve: A nonparametric approach. Biomentrics 44 $837-845,1988$

45. Zhou Q, Huang SX, Zhang F, Li SJ, Liu C, Xi YY, Wang L, Wang X, He QQ, Sun CC and Li DJ: MicroRNAs: A novel potential biomarker for diagnosis and therapy in patients with non-small cell lung cancer. Cell Prolif 50: e12394, 2017.

46. Belinsky SA: Gene-promoter hypermrthylation as a biomaker in lung cancer. Nat Rev Cancer 4: 707-717, 2004.

47. Liu X, Qu J, Xue W, He L, Wang J, Xi X, Liu X, Yin Y and Qu Y: Bioinformatics-based identification of potential microRNA biomarkers in frequent and non-frequent exacerbators of COPD. Int J Chron Obstruct Pulmon Dis 13: 1217-1228, 2018.

48. Kara M, Kirkil G and Kalemci S: Differential expression of microRNAs in chronic obstructive pulmonary disease. Adv Clin Exp Med 25: 21-26, 2016.

49. Hermann S, Brandes F, Kirchner B, Buschmann D, Borrmann M, Klein M, Kotschote S, Bonin M, Reithmair M, Kaufmann I, et al: Diagnostic potential of circulating cell-free microRNAs for comminity-acquired pneumonia and pneumonia-related sepsis. J Cell Mol Med 24: 12054-12064, 2020.

50. Yang DW, Zhang Y, Hong QY, Hu J, Li C, Pan BS, Wang Q, Ding FH, Ou JX, Liu FL, et al: Role of a serum-based biomarker panel in the early diagnosis of lung cancer for a cohort of high-risk patients. Cancer 121 (Suppl): S3113-S3121, 2015.

51. Kosaka N, Iguchi H, Yoshioka Y, Takeshita F, Matsuki Y and Ochiya T: Secretory mechanisms and intercellular transfer of microRNAs in living cells. J Boil Chem 285: 17442-17452, 2010.

52. Schwarzenbach H, Nishida N, Calin GA and Panted K: Clinical relevance of circulating cell-free microRNAs in cancer. Nat Rev Clin Oncol 11: 145-156, 2014.

53. Wang X, Zhi X, Zhang Y, An G and Feng G: Role of plasma microRNAs in the early diagnosis of non-small-cell lung cancer: A case-control study. J Thorac Dis 8: 1645-1652, 2016.

54. Xing L, Todd NW, Yu L, Fang H and Jiang F: Early detection of squamous cell lung cancer in sputum by a panel of microRNA markers. Mod Pathol 23: 1157-1164, 2010

55. Kim JO, Gazala S, Razzak R, Guo L, Ghosh S, Roa WH and Béard EL: Non-small cell lung cancer detection using microRNA expression profiling of bronchoalveolar lavage fluid and sputum. Anticancer Res 35: 1873-1880, 2015.

56. Zhang WC, Chi TM, Yang H, Nga ME, Lunny DP, Lim EK, Sun LL, Pang YH, Leow YN, Malusay SR, et al: Tumor-initiating cell-specific miR-1246 and miR-1290 expression converge to promote non-small cell lung cancer progression. Nat Commun 7: $11702,2016$.

57. Zhang L, Lin J, Ye Y, Oba T, Gentile E, Lian J, Wang J, Zhao Y, Gu J, Wistuba II, et al: Serum microRNA-150 predicts prognosis for early-stage non-small cell lung cancer and promotes tumor cell proliferation by targeting tumor suppressor gene SRCIN1. Clin Pharmacol Ther 103: 1061-1073, 2018.

58. Li H, Liu J, Cao W, Xiao X, Liang L, Liu-Smith F, Wang W, Liu H, Zhou P, Ouyang R, et al: C-myc/miR150/EPG5 axis mediated dysfunction of autophagy promotes development of non-small cell lung cancer. Theranostics 9: 5135-5148, 2019.

59. Li C, Yin Y, Liu X, Xi X, Xue W and Qu Y: Non-small cell lung cancer associated microRNA expression signature: Integrated bioinformatic analysis, valilation and clinical significance. Oncotarget 8: 24564-24578, 2017.

60. $\mathrm{Li} \mathrm{X}$ and Wu X: MiR-21-5P promotes the progression of non-small-cell lung cancer by regulating the expression of SMAD7. Onco Targets Ther 11: 8445-8454, 2018. 
61. Bertol G, Cava C and Castigliohi I: MicroRNAs: New biomarkers for diagnosis, prognosis, therapy prediction and therapeutic tools for breast cancer. Theranostics 5: 1122-1143, 2015.

62. Ahmed EK, Fahmy SA, Effat $\mathrm{H}$ and Wahab AHA: Circulating miR-210 and miR-1246 as potential biomarkers for differentiating hepatocellular carcinoma from metastatic tumors in the liver. J Med Biochem 38: 109-117, 2019.

63. Wei J, Yang L, Wu YN and Xu J: Serum miR-1290 and miR-1246 as potential diagnostic biomarkers of human pancreatic cancer. J Cancer 11: 1325-1333, 2020.

64. Kobayashi M, Sawada K, Nakamura K, Yoshimura A, Miyamoto M, Shimizu A, Ishida K, Nakatzuka E, Kodama M, Hashimoto K, et al: Exosomal miR-1290 is a potential biomarker of high-grade serous ovarian carcinoma and can disriminate patients from those with malignanies of other histologial types. J Ovarian Res 11: 81, 2018.
65. Sur D, Burz C, Sabarimurugan S and Irimie A: Diagnostic and prognostic significance of miR-150 in colorectal cancer: A systematic review and meta-analysis. J Pers Med 10: 99, 2020.

66. Kalogirou C, Ellinger J, Kristianse G, Hatzichristodoulou G, Kubler H, Kneitz B, Busch J and Fendler A: Identification of miR-21-5p and miR-210-3p serum levels as biomarkers for patients with papillary renal cell carcinoma: A multicenter analysis. Transl Androl Urol 9: 1314-1322, 2020.

67. Council for International Organizations of Medical Sciences: International ethical guidelines for biomedical research involving human subjects. Bull Med Ethics 182: 17-23, 2002.

68. World Medical Association: Declaration of Helsinki. 1964. http://www.wma.net/wp-content/uploads/2018/07/DoH-Jun1964. pdf.

69. World Medical Association: Declaration of Helsinki. 2008. https://www.wma.net/wp-content/uploads/2018/07/ DoH-Oct2008.pdf. 